\title{
Funding Innovative Healthcare Programs Through Social Impact Bonds: Issues and Challenges
}

\author{
Rosella Carè \\ University of Cagliari, Cagliari, Italy \\ Giuseppe Ferraro \\ University Magna Graecia, Catanzaro, Italy
}

\begin{abstract}
Social Impact Bonds (SIBs) have quickly attracted the attention of policy makers, practitioners, and academics due to their ability to fund innovative health and social programs while generating savings for governments and financial returns for investors. Health Impact Bonds (HIBs) represent the adaptation of these current examples of Social Impact Bonds in recidivism and child removal into new health programs and interventions that encourage investments in cost-saving preventive services in order to reduce the need for more costly remediation and fill the market gap between the private and public sectors. This work provides an overview of this emerging funding scheme and proposes reflections and suggestions useful for both practitioners and policy makers. Thus, the first part of the work presents an overview of the literature about SIBs and HIBs by highlighting the opportunities and challenges related to their implementation, the second part provides qualitative and quantitative data on all the existing HIBs, and the last part provides a discussion and suggestions useful for their implementation. ${ }^{1}$
\end{abstract}

Keywords: Social Impact Bonds (SIBs), healthcare programs, Health Impact Bonds (HIBs)

\section{Introduction}

The economic downturn has had a severe effect on the public finances of several countries and inevitably on their health systems (Karanikolos et al., 2013; Kentikelenis, Stubbs, \& King, 2015). This condition is common to all health system models and has potential consequences for healthcare supply and system performance (Keegan, Thomas, Normand, \& Portela, 2013). Defined as an emerging social policy instrument (Berndt \& Wirth, 2018) able to fund innovative health and social programs while generating profits for

\footnotetext{
Acknowledgements: Special thanks go to the Department of Law, Economics and Sociology of the University Magna Graecia of Catanzaro for granting opportunity to Rosella Carè to pursue her research project and of which this work is a result. Rosella Carè, Ph.D., Department of Economics and Business Administration, University of Cagliari, Cagliari, Italy.

Giuseppe Ferraro, Ph.D student, Department of Law, Economics and Sociology, University Magna Graecia, Catanzaro, Italy.

Correspondence concerning this article should be addressed to Rosella Carè, Department of Economics and Business Administration, University of Cagliari, Italy.

1 This work is the result of a collaboration between the authors. In particular, Ferraro contributed to the paragraph "Introduction" while Carè contributed to the following paragraphs: "Social Impact Bonds: How do they work?”; "Social Impact Bonds: A real win-win model?”; “A growing phenomenon around the globe: An overview of existing HIBs”; "Insights from a universal healthcare system: The case of the UK"; "Preventive programs to avoid future expenses: The case of the US"; "Promoting mental health through an innovative scheme of service provision: Evidence from Australia and New Zealand”; "Are HIBs the road to delivering the highest quality of care? Evidence From Canada”; "International capital to help populations in need of health assistance: Evidence from the first Humanitarian Impact Bond”; “Conclusions and future research directions”.
} 
investors and savings for governments (Rizzello \& Carè, 2016; Katz, Brisbois, Zerger, \& Hwang, 2018), Social Impact Bonds (SIBs) have quickly attracted the attention of policy makers, practitioners, and academics. The concept of SIB arose as a potential solution to problems faced by the social service sector, which suffers from a constant insufficiency of capital (Cox, 2011), by facilitating the private sector to become involved in the delivery of social services (Fox \& Albertson, 2012). SIBs can be used to enhance the transparency and evaluation of expenditures made by the public sector (Schinkus, 2017) and to monetize the benefits of social interventions. Although SIBs are not a miraculous way of financing welfare, they can significantly contribute to improving society by redesigning social programs through market-based solutions, enhancing transparency and the evaluation of government expenditures (Schinckus, 2017). The best candidates for this kind of private funding are programs with large upfront costs, programs that serve large numbers of people, and programs with a strong evidence base. Health Impact Bonds (HIBs) represent the adaptation of these current examples of Social Impact Bonds in recidivism and child removal into new health programs and interventions (Rowe \& Stephenson, 2016), encourage investments in cost-saving preventive services in order to reduce the need for more costly remediation (Clay, 2013; Fitzgerald, 2013; Galloway, 2014; Golden, 2014), and fill the market gap between the private and public sectors (Tan et al., 2015; Rowe \& Stephenson, 2016). Academic literature about HIBs shows two main trends: the first a tendency to underline the opportunities that this kind of innovative financial instrument offers for the provision of evidence-based programs (Rowe \& Stephenson, 2016; Katz et al., 2018), health promotion, and preventive medicine (R. S. Moran, D. S. Moran, \& Fire, 2018; Iovan \& Lantz, 2018), and the second is related to the evaluation and metrics aspects (Tan et al., 2015; Fraser, Tan, Lagarde, \& Mays, 2018). The review of the extant literature-including academic studies, government and private reports—-suggests the need for a systematic analysis of the benefits, costs, and risks in SIBs and HIBs contracts.

Despite the growing popularity of SIBs, very little research to date has explored their use, opportunities, and challenges in the healthcare sector. This work provides an overview on this emerging funding scheme and proposes reflections and suggestions useful for both practitioners and policy makers. Hence, the first part of the work presents an overview of the literature about SIBs and HIBs by highlighting the opportunities and challenges related to their implementation, while the second part provides qualitative and quantitative data on all the existing HIBs. The last part provides a discussion and suggestions useful for their implementation.

\section{Social Impact Bonds: How Do They Work?}

SIBs are not bonds as traditionally defined (Clifford \& Jung, 2016) but can be considered as an emerging model in which finance, service delivery and, supposedly, risk, are devolved from the public to the private sector. Following Sinclair, McHugh, Huckfield, Roy, and Donaldson (2014), the origin of SIBs dates back to the introduction of Payment-by-Result (PbR) schemes by the UK government in 2009. In particular, SIBs are conceived as a specific type of outcomes-based, or Payment by Results (PbR), contracting (Rizzello, Caridà, Trotta, Ferraro, \& Carè, 2018) in which all parties involved work collaboratively and flexibly rather than in classic public procurement schemes (Warner, 2013; Scognamiglio, Di Lorenzo, Sibillo, \& Trotta, 2018). Figure 1 shows the basic impact bond structure and the main types of actors that are usually involved.

The SIB scheme described in Figure 1 moves from a PPP approach for the provision of public services, based on an "optimal” risk-sharing and on an innovative design and delivery of public services (Carè, 2018). However, despite classic PPP schemes, SIBs involves private investors who materially fund the project and are then reimbursed if projects meet their objectives. The SIB starts when the outcome funder (e.g., governmental 
bodies, philanthropic or charitable entities) identifies a target population and a desired outcome and enters into a contract with the external delivery agent (Cooper, Graham, \& Himick, 2016; Clifford \& Jung, 2016). Generally, the outcome funder(s) (also called a "commissioner") is responsible for repaying the investors if the predetermined outcomes are reached. The contract between the commissioner and the external agency delivery sets out the outcomes which, if achieved, will activate the payments from the commissioner. The most critical piece involved in structuring the transaction is represented by the choice of the outcome metrics around which the impact bond contract is centered. Outcomes must be measurable and achievable within the scheduled time horizon. The external delivery agent-usually a financial intermediary or a social finance intermediary — markets the investment to the investors and is responsible for bringing the stakeholders together (Gustafsson-Wright, Gardiner, \& Putcha, 2015). The necessity of raising the funds or working capital required by the delivery agency to achieve the scheduled outcome(s) requires a further contract between the delivery agent and the socially motivated investors. Investors receive financial returns according to the social impact achieved and they do not obtain payback if the scheduled outcomes are not reached (Cooper et al., 2016; Scognamiglio et al., 2018). A further contract is established between the delivery agent and the service provider that will receive the working capital to provide the required services to the population in need. In addition to these four players, an external and independent evaluator measures, evaluates, and validates the outcome reached by the program. An experimental (e.g., randomized controlled trial), quasi-experimental, or nonexperimental design can be used to assess the program results (Gustafsson-Wright et al., 2015). The contractual structure described above enables the following: (i) the public sector (commissioner) to provide different kinds of welfare services and to share the risks with the private sector; (ii) the service provider(s) to benefit from increased flexibility in delivering the agreed-upon outcomes; and (iii) investors to finance activities designed to achieve positive social outcomes by providing the upfront capital (Carè, 2018).

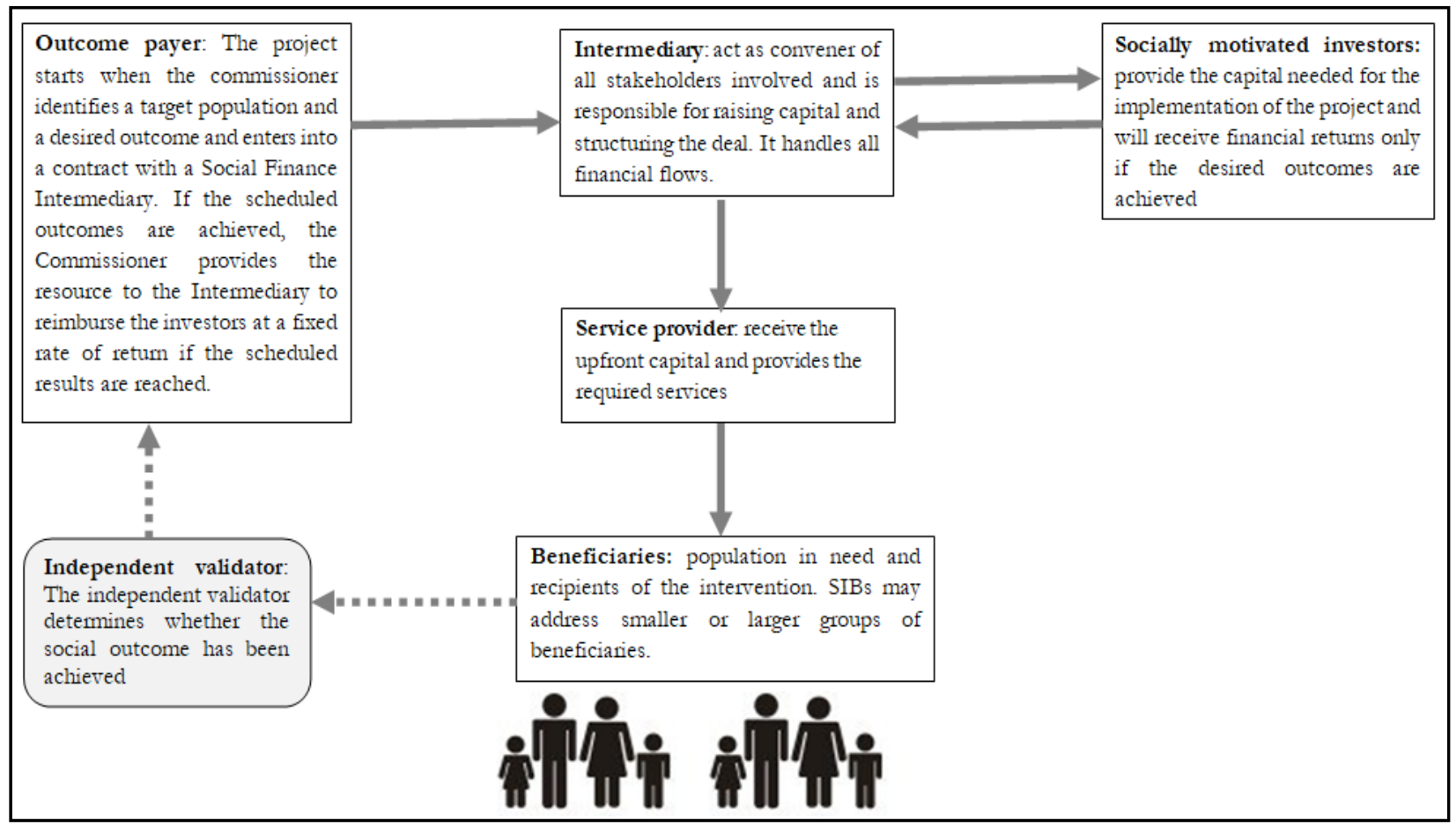

Figure 1. The Social Impact Bond mechanism. 


\section{Social Impact Bonds: A Real Win-Win Model?}

SIBs have spread rapidly across a range of public policy domains and in a number of countries, promoted with the idea of a "win-win" solution for governments, investors, service providers, and service users (Fraser et al., 2018). However, the academic literature has expressed several concerns about the structure of SIBs—and as a result of HIBs - from an accountability and governance perspective (McHugh, Sinclair, Roy, Huckfield, \& Donaldson, 2013; Warner, 2013; Fraser et al., 2018). Theoretically, these kinds of initiatives are able to expand accountability because programs are independently evaluated for their results, and the commissioner is required to pay the investors only if the program meets the scheduled metrics. Nevertheless, one could verify the hypothesis in which the program exceeds the metrics, and in this case the investor could receive an increased rate of return by making the social program more expensive for the public sector. From a governance perspective, McHugh et al. underline that

The loss of a direct relationship between service provider and government will enhance the build-up of asymmetric information in favour of the provider, and could reduce oversight and the ability of government to influence provision or step in if malpractice occurs. (2013, p. 251)

Moreover, in the SIB model, the desired goals of each party involved could differ from one to the other. In particular, by immediately receiving the upfront capital necessary for the delivery of the activities, the service provider could be less motivated than impact investors to achieve the scheduled outcomes. On the other hand, socially motivated investors receive their returns only after a rigorous evaluation of the outcomes achieved by an independent evaluator. At the same time, information asymmetries may occur between the service provider and both investors and commissioners in term of the appropriateness of outcome metric used for the evaluation of SIB success. Similarly, the risk aversion of the parties involved could be different for each actor. The transfer of risk is a primary objective in SIBs. The public sector partner seeks to divest itself of the risks associated with the delivery and operation of desired public facilities and services. Risk is central from the investor's perspective, as it determines the rate of return that investors require. From the commissioner's perspective, SIB represents a way to transfer the financial risk of a potentially unsuccessful policy to private parties. The SIBs' financial mechanisms determine the total bearing of the financial loss risk, in case of non-achievement of the outcome, by impact investors (Clifford \& Jung, 2016). Only in cases of SIB success will the public commissioner repay investors, which, on the contrary, presents a risk aversion in line with a "patient capital" investor profile. For such investors, risks are related to an investment timeline that produces a financial return in addition to a social return, generally achieved in the medium or long term. Several authors have tried to classify risks related to the SIB model (Emerson, 2012; Saltuk, 2012; Ng, Mirakhor, \& Ibrahim, 2015; Rizzello et al., 2018; Scognamiglio et al., 2018). Table 1 provides an overview of the main opportunities and challenges related to the implementation of an SIB initiative by considering all the relevant stakeholders that could be interested in the realization of this kind of project.

With regard to risks, Carè (2018) provides a classification of those that may occur in an SIB contract by distinguishing three main levels: (i) the macro level, which comprises regulatory or policy risks that may occur when new policies and new legislation are implemented, resulting in changes in the operating setting of the scheme; (ii) the meso level, which includes risks occurring within the boundaries of the project, as, for example programmatic (when the program does not work), operational (when the program is not executed as scheduled in the design phase), and evaluation risks (when errors occur in measuring results); (iii) and the micro level, 
which includes risks arising from the relationship between private and public actors (Rizzello et al., 2018). As more SIBs are launched, there is a need for independent evaluations of their outcomes and impacts (Jackson, 2013), and many authors have highlighted that the state of measurement of nonfinancial performance is weak (Saltuk, Bouri, Mudaliar, \& Pease, 2013; Reeder, Colantonio, Loder, \& Rocyn Jones, 2015). Flynn, Young, and Barnett (2015) and Wilson, Silva, and Ricardson (2015) underline the general scarcity of successful evaluation practices by noting the importance of identifying high-quality evaluation approaches. However, measurement is more than only metrics, and the development of a standard evaluation model should not leave out a clear understanding of what is important to measure for each stakeholder.

Table 1

Opportunities and Challenges in the SIB Model

\begin{tabular}{|c|c|c|}
\hline & Opportunities & Challenges \\
\hline Commissioner & $\begin{array}{l}\text { Public expenditure savings } \\
\text { More flexible and efficient service delivery } \\
\text { Better contract management } \\
\text { Better alignment of financial and social returns } \\
\text { External investment in the healthcare sector } \\
\text { Risk transfer } \\
\text { Performance monitoring and evaluation }\end{array}$ & $\begin{array}{l}\text { Stakeholder management/engagement } \\
\text { Scale up difficulties } \\
\text { Contractual complexity } \\
\text { Long time from the piloting stage to the } \\
\text { implementation and closing stage } \\
\text { Lack of retail investors } \\
\text { Performance monitoring and evaluation }\end{array}$ \\
\hline $\begin{array}{l}\text { Socially motivated } \\
\text { investors }\end{array}$ & $\begin{array}{l}\text { Investment diversification } \\
\text { Alignment of social and financial returns } \\
\text { Positive and measurable social impact }\end{array}$ & $\begin{array}{l}\text { Policy uncertainty } \\
\text { Absence of track records and of standardized models } \\
\text { Long time from the piloting stage to the } \\
\text { implementation and closing stage } \\
\text { Financial risks } \\
\text { Absence of divestment opportunities }\end{array}$ \\
\hline $\begin{array}{l}\text { Philanthropists and } \\
\text { charitable } \\
\text { foundations }\end{array}$ & $\begin{array}{l}\text { Possibility of investing and reinvesting their capital } \\
\text { instead of always providing irrevocable donations or } \\
\text { grants } \\
\text { Positive social impact } \\
\text { Positive and measurable social impact }\end{array}$ & $\begin{array}{l}\text { Policy uncertainty } \\
\text { Absence of track records and of standardized models } \\
\text { Long time from the piloting stage to the } \\
\text { implementation and closing stage } \\
\text { Financial risks } \\
\text { Absence of divestment opportunities }\end{array}$ \\
\hline $\begin{array}{l}\text { External delivery } \\
\text { agency }\end{array}$ & New market opportunities & $\begin{array}{l}\text { Long time from the piloting stage to the } \\
\text { implementation and closing stage } \\
\text { Rigid contractual boundaries }\end{array}$ \\
\hline Service provider & $\begin{array}{l}\text { Upfront capital and immediate access to the financial } \\
\text { resource needed } \\
\text { New market opportunities }\end{array}$ & $\begin{array}{l}\text { Innovation in program delivery } \\
\text { Rigid contractual boundaries }\end{array}$ \\
\hline Population in need & $\begin{array}{l}\text { Out-of-pocket or innovative services } \\
\text { Better outcomes }\end{array}$ & Experimental level of the programs \\
\hline $\begin{array}{l}\text { Tax } \\
\text { payers/community }\end{array}$ & $\begin{array}{l}\text { Public expenditure savings } \\
\text { Accountability } \\
\text { Societal benefit through positive } \\
\text { Impact }\end{array}$ & Performance monitoring and evaluation \\
\hline
\end{tabular}

\section{A Growing Phenomenon Around the Globe: An Overview of Existing HIBs}

During the last few years, many SIBs have been launched and have emerged as a widely hailed innovation (Clifford \& Jung, 2016). The first SIB was launched in 2010 - in the aftermath of the economic crisis—and quickly picked up internationally and across different sectors (Clifford \& Jung, 2016). SIBs are being initiated by city administrations and local or regional authorities, but the majority has been conceived and launched by national or state governments (OECD, 2016). According to the Social Finance Database, thus far 121 SIBs have been launched and more than 70 are being developed, with a total amount of raised capital of $\$ 413$ million. 
SIBs are actually available in Australia (8), Austria (1), Belgium (1), Canada (4), Cameroon (1), Congo (1), Colombia (1), Germany (3), Finland (2), France (2), Japan (3), India (3), Israel (2), the Netherlands (8), New Zealand (1), Peru (1), Portugal (4), South Africa (1), South Korea (2), Sweden (1), Switzerland (1), Uganda (1), the UK (47), and the US (22) (Social Finance, 2018).

SIBs target social issues such as criminal justice (11), homelessness (23), child and family welfare (14), early childhood education (11), workforce development (37), health (22), poverty and environments (2), and adults with complex needs (1) (Social Finance, 2018). Currently, the 22 HIBs launched have raised 90.6 million dollars (Social Finance, 2018) and are available in both developed and undeveloped countries as Humanitarian Impact Bonds (Nigeria, Mali, and the Democratic Republic of Congo) or Development Impact Bonds (Cameroon) (Table 2).

Table 2

HIBs Launched

\begin{tabular}{|c|c|c|c|c|c|c|c|}
\hline SIB name & Country & $\begin{array}{l}\text { Healthcare } \\
\text { issues }\end{array}$ & $\begin{array}{l}\text { Target } \\
\text { population }\end{array}$ & $\begin{array}{l}\text { Launch } \\
\text { date }\end{array}$ & $\begin{array}{l}\text { Capital } \\
\text { raised }\end{array}$ & Investors & Outcomes founder \\
\hline $\begin{array}{l}\text { Mental Health } \\
\text { and Employment } \\
\text { Social Impact } \\
\text { Bond }\end{array}$ & $\begin{array}{l}\text { New } \\
\text { Zealand }\end{array}$ & $\begin{array}{l}\text { Mental health } \\
\text { and } \\
\text { employment }\end{array}$ & $\begin{array}{l}1,700 \text { people } \\
\text { with a } \\
\text { diagnosed } \\
\text { mental health } \\
\text { condition }\end{array}$ & $\begin{array}{l}\text { February } \\
2017\end{array}$ & $\begin{array}{l}\mathrm{N} \$ 1.5 \\
\text { million }\end{array}$ & $\begin{array}{l}\text { 1) APM Workcare } \\
\text { 2) Janssen } \\
\text { 3) Prospect Investment } \\
\text { Management Limited } \\
\text { 4) Wilberforce } \\
\text { Foundation }\end{array}$ & $\begin{array}{l}\text { New Zealand } \\
\text { Ministry of Social } \\
\text { Development }\end{array}$ \\
\hline $\begin{array}{l}\text { Community } \\
\text { Hypertension } \\
\text { Prevention } \\
\text { Initiative }\end{array}$ & Canada & Hypertension & $\begin{array}{l}7,000 \\
\text { prehypertensive } \\
\text { older adults } \\
(60+) \text { in } \\
\text { Toronto and } \\
\text { Vancouver }\end{array}$ & $\begin{array}{l}\text { October } \\
2016\end{array}$ & $\mathrm{C} \$ 2 \mathrm{M}$ & $\begin{array}{l}\text { Foundations, } \\
\text { high-net-worth } \\
\text { individuals, and } \\
\text { companies }\end{array}$ & $\begin{array}{l}\text { Public Health } \\
\text { Agency of Canada }\end{array}$ \\
\hline $\begin{array}{l}\text { The Cameroon } \\
\text { Cataract } \\
\text { Development } \\
\text { Impact Bond }\end{array}$ & Cameroon & $\begin{array}{l}\text { Blindness and } \\
\text { vision } \\
\text { impairment }\end{array}$ & $\begin{array}{l}18,000 \\
\text { people in } \\
\text { Cameroon in } \\
\text { need of cataract } \\
\text { eye surgery }\end{array}$ & $\begin{array}{l}\text { October } \\
2017\end{array}$ & $\$ 2.5 \mathrm{M}$ & $\begin{array}{l}\text { 1) Overseas Private } \\
\text { Investment } \\
\text { Corporation (OPIC) } \\
(87.5 \%) \\
\text { 2) The Netri } \\
\text { Foundation (12.5\%) }\end{array}$ & $\begin{array}{l}\text { 1) The Conrad N. } \\
\text { Hilton Foundation } \\
(80 \%) \\
\text { 2) The Fred } \\
\text { Hollows } \\
\text { Foundation }(10 \%) \\
\text { 3) Sightsavers } \\
(10 \%)\end{array}$ \\
\hline $\begin{array}{l}\text { Preventing Type } \\
\text { II Diabetes }\end{array}$ & Israel & $\begin{array}{l}\text { Diabetes } \\
\text { prevention }\end{array}$ & $\begin{array}{l}2,250 \text { people at } \\
\text { risk of } \\
\text { developing } \\
\text { Type } 2 \\
\text { Diabetes }\end{array}$ & $\begin{array}{l}\text { March } \\
2016\end{array}$ & $\$ 5.5 \mathrm{M}$ & $\begin{array}{l}\text { Multiple investors } \\
\text { (coordinated by UBS } \\
\text { banking corporation) }\end{array}$ & $\begin{array}{l}\text { 1) Israeli Health } \\
\text { Maintenance } \\
\text { Organisations } \\
\text { (Clalit \& Leumit) } \\
\text { 2) National } \\
\text { Insurance Institute }\end{array}$ \\
\hline $\begin{array}{l}\text { The Utkrisht } \\
\text { Impact Bond }\end{array}$ & India & $\begin{array}{l}\text { Maternal and } \\
\text { neonatal } \\
\text { mortality }\end{array}$ & $\begin{array}{l}\text { Up to } 600,000 \\
\text { pregnant } \\
\text { women in the } \\
\text { state of } \\
\text { Rajasthan }\end{array}$ & $\begin{array}{l}\text { November } \\
2017\end{array}$ & $\$ 3.5 \mathrm{M}$ & $\begin{array}{l}\text { 1) UBS Optimus } \\
\text { Foundation } \\
\text { 2) Implementing } \\
\text { Partners }\end{array}$ & $\begin{array}{l}\text { 1) USAID } \\
\text { 2) Merck for } \\
\text { Mothers } \\
\text { 3) Government of } \\
\text { Rajasthan }\end{array}$ \\
\hline $\begin{array}{l}\text { South Carolina } \\
\text { Nurse-Family } \\
\text { Partnership Pay } \\
\text { for Success } \\
\text { Project }\end{array}$ & US & $\begin{array}{l}\text { Early childhood } \\
\text { development }\end{array}$ & $\begin{array}{l}3,200 \\
\text { first-time, } \\
\text { low-income } \\
\text { mothers over } \\
\text { four years }\end{array}$ & $\begin{array}{l}\text { February } \\
2016\end{array}$ & $\$ 30 \mathrm{M}$ & $\begin{array}{l}\text { 1) The Duke } \\
\text { Endowment } \\
\text { 2) BlueCross } \\
\text { BlueShield of South } \\
\text { Carolina Foundation } \\
\text { 3) The Boeing } \\
\text { Foundation } \\
\text { 4) Laura and John } \\
\text { Arnold Foundation }\end{array}$ & $\begin{array}{l}\text { 1) Government of } \\
\text { South Carolina }\end{array}$ \\
\hline
\end{tabular}


(Table 2 to be continued)

\begin{tabular}{|c|c|c|c|c|c|c|c|}
\hline & & & & & & $\begin{array}{l}\text { 5) Greenville County } \\
\text { SC First Steps } \\
\text { 6) Medicaid } \\
\text { 7) Private funders }\end{array}$ & \\
\hline $\begin{array}{l}\text { The Blood Bank } \\
\text { of Delmarva } \\
\text { Young Blood } \\
\text { Sustainability } \\
\text { Project }\end{array}$ & US & $\begin{array}{l}\text { Recruiting new } \\
\text { blood donors }\end{array}$ & $\begin{array}{l}\text { Potential blood } \\
\text { donors in } \\
\text { Delaware }\end{array}$ & $\begin{array}{l}\text { August } \\
2018\end{array}$ & $\$ 0.45 \mathrm{M}$ & $\begin{array}{l}\text { DCF Social Impact } \\
\text { Fund }\end{array}$ & $\begin{array}{l}\text { Longwood } \\
\text { Foundation }\end{array}$ \\
\hline $\begin{array}{l}\text { Strong } \\
\text { Beginnings Pay } \\
\text { for Success } \\
\text { Project }\end{array}$ & US & $\begin{array}{l}\text { High-risk } \\
\text { pregnant } \\
\text { women, with } \\
\text { additional } \\
\text { services for } \\
\text { children } \\
\text { extending from } \\
\text { infants to age 2 } \\
\end{array}$ & $\begin{array}{l}1,700 \text { high-risk } \\
\text { pregnant } \\
\text { women served } \\
\text { by Medicaid, } \\
\text { and their } \\
\text { families }\end{array}$ & $\begin{array}{l}\text { August } \\
2016\end{array}$ & $\$ 8.5 \mathrm{M}$ & $\begin{array}{l}\text { W.K. Kellogg } \\
\text { Foundation; } \\
\text { Federal Human } \\
\text { Services } \\
\text { Administration; } \\
\text { Spectrum Health; } \\
\text { Michigan Health } \\
\text { Endowment Fund }\end{array}$ & State of Michigan \\
\hline $\begin{array}{l}\text { Mental Health } \\
\text { and Employment } \\
\text { Partnership }\end{array}$ & UK & $\begin{array}{l}\text { Mental health } \\
\text { and } \\
\text { employment }\end{array}$ & $\begin{array}{l}\text { 2,500 people } \\
\text { with severe } \\
\text { mental illness } \\
\text { (typically with } \\
\text { a diagnosis of } \\
\text { psychosis, such } \\
\text { as } \\
\text { schizophrenia, } \\
\text { bipolar disorder, } \\
\text { or severe } \\
\text { depression or } \\
\text { anxiety) } \\
\text { currently in } \\
\text { contact with } \\
\text { statutory } \\
\text { mental health } \\
\text { services. }\end{array}$ & $\begin{array}{l}\text { January } \\
2016\end{array}$ & $£ 0.4 \mathrm{M}$ & Big Issue Invest & $\begin{array}{l}\text { Cabinet Office } \\
\text { Social Outcomes } \\
\text { Fund; } \\
\text { Haringey Council } \\
\text { and CCG; } \\
\text { Tower Hamlets } \\
\text { CCG; } \\
\text { Staffordshire } \\
\text { County Council } \\
\text { and CCGs }\end{array}$ \\
\hline $\begin{array}{l}\text { Your Life Line } \\
24 / 7\end{array}$ & UK & End of life care & $\begin{array}{l}\text { Adults over the } \\
\text { age of } 18 \text { who } \\
\text { are in the last } \\
\text { weeks of life } \\
\text { and registered } \\
\text { with a } \\
\text { Hillingdon G.P. }\end{array}$ & $\begin{array}{l}\text { September } \\
2018\end{array}$ & n.a & $\begin{array}{l}\text { Care and Wellbeing } \\
\text { Fund }\end{array}$ & Hillingdon CCG \\
\hline $\begin{array}{l}\text { Elton John AIDS } \\
\text { Foundation } \\
\text { (unofficial name) }\end{array}$ & UK & $\begin{array}{l}\text { HIV prevention } \\
\text { and treatment }\end{array}$ & $\begin{array}{l}\text { HIV high risk } \\
\text { groups and } \\
\text { HIV patients in } \\
\text { South London }\end{array}$ & $\begin{array}{l}\text { December } \\
2017\end{array}$ & n.a & $\begin{array}{l}\text { 1) Impact investing } \\
\text { funds } \\
\text { 2) Private investors } \\
\text { 3) Elton John AIDS } \\
\text { Foundation }\end{array}$ & $\begin{array}{l}\text { 1) Lambeth } \\
\text { 2) Lewisham and } \\
\text { Southwark CCGs } \\
\text { 4) NHS England } \\
\text { 5) Big Lottery } \\
\text { Fund } \\
\end{array}$ \\
\hline $\begin{array}{l}\text { Positive } \\
\text { Behavioral } \\
\text { Support (PBS) in } \\
\text { Bradford }\end{array}$ & UK & $\begin{array}{l}\text { Learning } \\
\text { disabilities in } \\
\text { children }\end{array}$ & $\begin{array}{l}\text { 14 Children } \\
\text { aged } 8 \text { to } 14 \\
\text { with learning } \\
\text { disabilities } \\
\text { and/or autism } \\
\text { and behavior } \\
\text { that challenges } \\
\text { - who are } \\
\text { likely to enter } \\
\text { residential care } \\
\text { in the medium } \\
\text { term }\end{array}$ & $\begin{array}{l}\text { November } \\
2017\end{array}$ & n.a & $\begin{array}{l}\text { Social and Sustainable } \\
\text { Capital (SASC) }\end{array}$ & $\begin{array}{l}\text { 1) Bradford City } \\
\text { Council } \\
\text { 2) Big Lottery } \\
\text { Fund }\end{array}$ \\
\hline
\end{tabular}


(Table 2 to be continued)

\begin{tabular}{|c|c|c|c|c|c|c|c|}
\hline Healthier Devon & UK & $\begin{array}{l}\text { Diabetes } \\
\text { prevention }\end{array}$ & $\begin{array}{l}\text { Prediabetic } \\
\text { adults with a } \\
\text { focus on } 40 \% \\
\text { most deprived } \\
\text { population } \\
\end{array}$ & June 2018 & n.a. & $\begin{array}{l}\text { Bridges Fund } \\
\text { Management }\end{array}$ & $\begin{array}{l}\text { Commissioning } \\
\text { Better Outcomes } \\
\text { Fund (Big Lottery } \\
\text { Fund) }\end{array}$ \\
\hline Reconnections & UK & Social isolation & $\begin{array}{l}\text { At least 3,000 } \\
\text { people aged } 50 \\
\text { years and over } \\
\text { classified on } \\
\text { the UCLA } \\
\text { loneliness scale } \\
\text { (a common } \\
\text { loneliness } \\
\text { measure) as } 8 \\
\text { to } 12 \text { (though it } \\
\text { is expected that } \\
\text { most clients } \\
\text { will be } 65+\text { ) }\end{array}$ & July 2015 & $£ 0.85 \mathrm{M}$ & $\begin{array}{l}\text { 1) Care and Wellbeing } \\
\text { Fund } \\
\text { 2) Nesta } \\
\text { 3) Impact Investments } \\
\text { Age UK }\end{array}$ & $\begin{array}{l}\text { 1) Cabinet Office } \\
\text { Social Outcomes } \\
\text { Fund } \\
\text { 2) Redditch \& } \\
\text { Bromsgrove CCG } \\
\text { 3) South } \\
\text { Worcestershire } \\
\text { CCG } \\
\text { 4) Worcestershire } \\
\text { County Council } \\
\text { 5) Wyre Forest } \\
\text { CCG }\end{array}$ \\
\hline Ways to Wellness & UK & $\begin{array}{l}\text { Social } \\
\text { prescribing }\end{array}$ & $\begin{array}{l}11,000 \text { people } \\
\text { with long-term } \\
\text { health } \\
\text { conditions such } \\
\text { as lung disease, } \\
\text { diabetes and } \\
\text { asthma }\end{array}$ & $\begin{array}{l}\text { March } \\
2015\end{array}$ & $£ 1.7 \mathrm{M}$ & Bridges Ventures & $\begin{array}{l}\text { Big Lottery } \\
\text { Commissioning } \\
\text { Better Outcomes } \\
\text { Fund; } \\
\text { Cabinet Office } \\
\text { Social Outcomes } \\
\text { Fund; } \\
\text { Newcastle West } \\
\text { Clinical } \\
\text { Commissioning } \\
\text { Group }\end{array}$ \\
\hline $\begin{array}{l}\text { End of Life Care } \\
\text { Integrator \& The } \\
\text { Advance Care } \\
\text { Plan Facilitator } \\
\text { (unofficial name) }\end{array}$ & UK & End-of-life care & $\begin{array}{l}\text { Adult residents } \\
\text { of the care } \\
\text { homes in their } \\
\text { last year of life }\end{array}$ & $\begin{array}{l}\text { April } \\
2017\end{array}$ & n.a. & $\begin{array}{l}\text { Care and Wellbeing } \\
\text { Fund }\end{array}$ & Haringey CCG \\
\hline $\begin{array}{l}\text { Resolve Social } \\
\text { Benefit Bond }\end{array}$ & Australia & $\begin{array}{l}\text { Severe mental } \\
\text { health issues }\end{array}$ & $\begin{array}{l}\text { Approximately } \\
530 \text { adults who } \\
\text { have been } \\
\text { hospitalized for } \\
\text { between } 40 \text { and } \\
270 \text { days, with } \\
\text { at least one } \\
\text { admission as a } \\
\text { mental health } \\
\text { inpatient, in the } \\
\text { previous } 12 \\
\text { months }\end{array}$ & June 2017 & AUD7 M & TBC & $\begin{array}{l}\text { The Government } \\
\text { of NSW acting } \\
\text { through the Health } \\
\text { Administration } \\
\text { Corporation } \\
\text { (HAC) }\end{array}$ \\
\hline $\begin{array}{l}\text { The Cancer and } \\
\text { Work Health } \\
\text { Impact Bond } \\
\end{array}$ & Netherlands & $\begin{array}{l}\text { Rehabilitation } \\
\text { of cancer } \\
\text { survivors } \\
\end{array}$ & $\begin{array}{l}140 \text { cancer } \\
\text { survivors }\end{array}$ & $\begin{array}{l}\text { November } \\
2017\end{array}$ & $€ 0.64 \mathrm{M}$ & $\begin{array}{l}\text { ABN AMRO Social } \\
\text { Impact Fund; } \\
\text { Start Foundation } \\
\end{array}$ & De Amersfoortse \\
\hline $\begin{array}{l}\text { The Program for } \\
\text { Humanitarian } \\
\text { Impact Investing } \\
\text { (PHII) }\end{array}$ & $\begin{array}{l}\text { Congo, } \\
\text { Mali, } \\
\text { Nigeria }\end{array}$ & $\begin{array}{l}\text { Physical } \\
\text { disabilities }\end{array}$ & $\begin{array}{l}\text { People with } \\
\text { disabilities in } \\
\text { conflict-hit } \\
\text { countries }\end{array}$ & $\begin{array}{l}\text { September } \\
2017\end{array}$ & $\$ 27 \mathrm{M}$ & $\begin{array}{l}\text { Investors from } \\
\text { Lombard Odier Bank }\end{array}$ & $\begin{array}{l}\text { La Caixa } \\
\text { Foundation } \\
\text { DFID } \\
\text { SECO } \\
\text { LIFT } \\
\end{array}$ \\
\hline
\end{tabular}




\section{Insights From a Universal Healthcare System: The Case of the UK}

With eight HIBs launched, the United Kingdom covers 36\% of the entire market. The first HIB-Ways to Wellness-was launched in 2015 and is focused on people with long-term conditions that represent the most frequent users of UK healthcare services. Treatment and care of those with long-term conditions accounts for 70 percent of the primary and acute care budget in England (GO Lab, 2017). In this vein, Ways to Wellness SIB adds to and complements medical support through social prescribing. Patients are helped to manage their long-term conditions through one-to-one support and given access to community-based activities such as becoming more active, learning to eat and cook more healthily, or getting back to work. The program was commissioned by the Newcastle Gateshead Clinical Commissioning Group, which will pay up to $£ 8.2 \mathrm{~m}$ to the service providers based on the achievement of two outcomes: improved self-management of LTC and reduced costs of secondary healthcare services. The service provider is receiving an upfront investment of $£ 1.65 \mathrm{~m}$ from the Bridges' Social Sector Funds, to be repaid in the later years of the project (CBOFE, 2014). The success of the program will be measured by an improvement in patient wellbeing and reductions in hospital visits, hospital admissions, and the length of hospital stays (Bridges Ventures, 2015; CBOFE, 2014; CBOFE, 2016a). The second HIB implemented—named The Mental Health and Employment Social Impact Bond (SIB)—will provide support to approximately 2,500 individuals living with severe mental health illness over three years to help them achieve competitive, paid employment. The SIB has been developed under the Mental Health and Employment Partnership (MHEP)—a social purpose company — that raised a pool of socially motivated investments from Big Issue Invest to finance service provision upfront, with investors repaid with up to $£ 1.3 \mathrm{~m}$ of outcomes-based Cabinet Office funding when user engagement, job entry, and job sustainment outcomes are achieved (CBOFE, 2016b). The Reconnections Social Impact Bond aims to reduce loneliness and isolation for 3,000 people over the age of 50 in Worcestershire. The Worcestershire County Council (WCC) and three cocommissioners from Clinical Commissioning Groups commissioned this SIB, with Nesta as the main funder and Age UK Herefordshire and Worcestershire the main service provider (CBOFE, 2016a). Though the development of this SIB, savings of $£ 3$ million to the public purse over a 15 -year period are estimated, while the costs of development were around £189,000. Between 2017 and 2018, five new HIBs have been launched, with programs spanning end-of-life care (Your Life Line 24/7) and HIV prevention and treatment (launched by the Elton John AIDS Foundation) to learning disabilities (Positive Behavioural Support (PBS) in Bradford) and diabetes prevention (Healthier Devon).

\section{Preventive Programs to Avoid Future Expenses: The Case of the US}

Moving from the first experiences in the UK, other countries have explored the use of HIBs in promoting healthcare programs. In 2015, in the United States, New Jersey established a \$15-million loan program to reduce public health costs by attracting private investment to preventive and early intervention care (CNCS, 2016). The project moves from the consideration that currently the US spends more than 15 percent of its gross domestic product, or $\$ 2.7$ trillion a year, on healthcare costs. Through an SIB initiative, the government can reduce its expenditures on treatment, hospitalization, and medical care (Barajas et al., 2014). To date, state level SIB activity has focused on legislative efforts to authorize the process, create study committees, begin pilot projects, engage in feasibility studies, and learn which types of programs this financing tool can be effectively used for (CNCS, 2016). In this vein, examples include Colorado, Idaho, Texas, 
New Hampshire, New Jersey, Pennsylvania, and Washington, which have introduced legislation related to SIBs since 2015. In 2016, South Carolina launched the nation's first HIBs focused on improving health outcomes for mothers and children living in poverty. Nurse-Family Partnership pairs vulnerable mothers with specially trained nurses who support mothers to undergo healthy pregnancies and become knowledgeable and responsible parents (ICS, 2016). The success of this pay-for-success initiative is measured through four metrics: (i) reduction in preterm births; (ii) reduction in child hospitalization and emergency department usage due to injury; (iii) increase in healthy spacing between births; and (iv) increased number of first-time mothers served in predetermined ZIP codes with high concentrations of poverty (ICS, 2016; Normile, VanLandeghem, \& King, 2017). As for the financial terms, philanthropic funders have committed \$17 million of the total $\$ 30$ million mobilized for the project, while Medicaid will fund approximately $\$ 13$ million. South Carolina will make up to $\$ 7.5 \mathrm{M}$ in success payments to sustain the service if evaluators find positive results (ICS, 2016).

\section{Promoting Mental Health Through an Innovative Scheme of Service Provision: Evidence From Australia and New Zealand}

In 2013, New South Wales pioneered Australia's first two SIBs to improve outcomes for children and families involved with child protection services: the Newpin Social Benefit Bond and The Benevolent Society Social Benefit Bond. New South Wales (NSW) has a clear commitment to supporting the broader social impact investment market to develop new and innovative ways to deliver services to individuals and communities (Dear, Helbitz, Khare, Lotan, Newman, Sims, \& Zaroulis, 2016). In February 2015, a Social Impact Investment Policy was launched outlining the State's aim to deliver two new social impact investments to market each year (OECD, 2016). The Resolve SBB provides Investors with the opportunity to fund a program that aims to improve the mental health and wellbeing of hundreds of individuals in NSW, while earning a financial return on their investment. Between 2012 and 2015, there were approximately 2,000 individuals each year spending between 40 and 270 days as mental health inpatients in NSW. Social Ventures Australia has partnered with Flourish Australia and the NSW Government to develop the Resolve Program aimed at improving the lives of people with mental health issues (Trotta, Caré, Severino, Migliazza, \& Rizzello, 2015). The program combines a residential service for periodic intensive support; integrated psychosocial, medical, and mental health support; and a warm line for after-hours support from peers. Investor returns are linked to outcome payments made by the NSW Government to the Resolve SBB Trust, which are based on government savings generated by the Resolve Program (Rizzello et al., 2018). Government savings are measured by comparing participants' consumption of health services with that of a control group. Moving from the consideration that a job improves individual health and wellbeing, the Mental Health and Employment Social Impact Bond-the first HIB developed in New Zealand-will help approximately 1,700 people with mental illness to achieve paid employment. The bond, which will run for six years, is backed by four investors, including a philanthropist (Wilberforce Foundation), a pharmaceutical company (Janssen), and an investment banking firm (Prospect Investment Management Ltd.), as well as APM (an employment and social care organization). The aim of the SIB is to get 1,700 people in South Auckland with mental health conditions into work over the next five years. Returns for investors will be tied to the number of workers placed and on how long they remain employed (Rizzello et al., 2018). The social impact target is to get $43 \%$ of the people it deals with into work, compared with a success rate of 30\% in other contracts the government currently operates. The $\$ 1.5$ million bond has 
been broken up into two tranches: $\$ 1.2$ million with an annual 7 percent return on the base case of placement rate of 43 percent, and a $\$ 300,000$ tranche paying 13 percent on the base case.

\section{Are HIBs the Road to Delivering the Highest Quality of Care? Evidence From Canada}

When compared with other high-income countries, Canada's health system performs poorly, does not deliver the highest quality of care to citizens, and does not deliver good value for money (Wise, 2017). Outcomes-funding arrangements offer governments a range of potential benefits. For these reasons, in October 2016, the Public Health Agency of Canada launched the country's first social impact bond in preventative health (Wise, 2017). The project, in collaboration with the MaRS Discovery District and Heart and Stroke Foundation, will use an outcomes-payment approach to fund a lifestyle change program, the Community Hypertension Prevention Initiative (CHPI), to prevent prehypertensive seniors progressing to full hypertension (Rizzello et al., 2018). The CHPI was offered in communities across Toronto starting in June 2017 and Vancouver starting in October 2018. The initiative will enroll 7,000 prehypertensive Canadians in a six-month program to help them adopt healthy behaviors and control their blood pressure. The bond was structured by the MaRS Centre for Impact Investing and 11 investors have invested.

\section{International Capital to Help Populations in Need of Health Assistance: Evidence From the First Humanitarian Impact Bond}

The world's first Humanitarian Impact Bond has been launched in collaboration with the International Committee of the Red Cross (ICRC) for the delivery of physical rehabilitation services in postconflict countries. The financial resources will be used to build and run three new physical rehabilitation centers in Africa (Congo, Mali, and Nigeria). At the end of the program, in 2022, the five donors participating in the program (the governments of Belgium, Switzerland, Italy, and the UK, and the Spanish "la Caixa” Foundation) will pay the ICRC an amount which is contingent on the efficiency of the three centers and that will be verified by an independent auditor using a benchmark specified in the contract. The ICRC will receive the payment if the level of efficiency is higher than the benchmark; if, instead, the performance is below the benchmark, the investors will lose part of their capital (Monnet \& Panizza, 2017). The Belgian government has already pledged $€ 10$ million to the bond in support of the ICRC's program (GHA, 2017). From a financial point of view, the structure of this first Humanitarian Impact Bond is based on a "pay-for-success" mechanism whereby investors provide upfront capital for the intervention, and the outcome founders (DFID, SECO, LIFT, and La Caixa Foundation) repay this capital along with a financial return of up to $7 \%$ if the bond reaches or outperforms the benchmark. If this fails to happen, investors could lose up to $40 \%$ of their capital (Alderson, 2018). Outcome payments will increase in line with improvements in the efficiency ratio, and interest payments are capped at a $7 \%$ internal rate of return.

\section{Conclusions and Future Research Directions}

This paper has sought to provide an overview of HIBs as an emerging funding model for the healthcare sector from both theoretical and practical perspectives. Rooted in the New Public Management approach, SIBs emerged in the aftermath of the economic and financial crisis as a response to the severe cuts experienced by social programs. SIBs are one of the most important talked-about instruments in the public delivery of welfare services landscape. Many important experiences are currently available all over the world. The United 
Kingdom is the country with the highest number of HIBs launched. This is the result of the development of a governmental policy—based on a pay-for-performance approach—for the provision of services to people generally not served in a universal health system. This is the case, for example, for people at the end of their life and for whom dying in their usual place of residence and having fewer emergency hospital admissions in their last month of life could be provided outside the traditional health system scheme by guaranteeing not only cost savings (through the reduction of hospitalization costs) but also improving quality and patient experience. Moreover, the UK experience shows that the development of an SIB requires not only a governmental policy but also a social investment environment comprising both investors and dedicated social finance intermediaries. The development of the HIB market also encourages the entry into the healthcare landscape of new players such as financial giants, social finance intermediaries, philanthropic institutions, and social enterprises. The number of HIB experiences around the world is growing. Governments are trying to develop their own schemes and contracts and consider HIBs a viable way to fund out-of-pocket programs. The Health Impact Bonds currently developed promote preventive programs with the promise of future public expenditure savings and have been developed in all three extant types of healthcare payment systems: single-payer system (e.g., the UK), two-tier system (e.g., Australia and New Zealand), and insurance mandate system (e.g., the United States). Currently, HIBs are active in many countries and in many areas of health intervention. The literature overview provided has tried to underline the main aspects now under the magnifying glass of academics and practitioners. First, the questions surrounding the right balance between opportunities and challenges. From a theoretical point of view, HIBs are able to provide governments with consistent savings. The public sector could be attracted by the idea of providing welfare services in areas difficult to reach using the standard approach combined with cost-savings, accountability of taxpayer funds, and value-for-money. In this sense, the overall "benefit" or "social benefits" of a social program designed using an SIB model is not necessarily a mere cost-saving benefit, but could also be understood in terms of added value, increased income, and externalities.

A key challenge for government is to find a rigorous and feasible method of evaluating whether SIBs can provide a better approach to delivering welfare services than traditional methods. Lastly, future research could be directed at an analysis of aspects such as information asymmetries between the involved parties and of the entire set of risks emerging from the contractual schemes used in the HIB model.

\section{References}

Albertson, K., \& Fox, C. (2018). Payment by results and Social Impact Bonds: Outcome-based payment systems in the UK and US. Bristol, England: Policy Press.

Alderson, H. (2018). How the first Humanitarian Impact Bond will transform financing of aid, bond. Retrieved from https://www.bond.org.uk/news/2018/02/how-the-first-humanitarian-impact-bond-will-transform-financing-of-aid

Arena, M., Bengo, I., Calderini, M., \& Chiodo, V. (2016). Social Impact Bonds: Blockbuster or flash in a pan? International Journal of Public Administration, 39(12), 927-939.

Barajas, A., Burt, K., Johnson, P., Licona, J., Parker, W., Sturtevant, L., ... \& Yeh, C. (2014). Social Impact Bonds. A new tool for $\begin{array}{llll}\text { social financing. } & \text { Princeton } & \text { University. }\end{array}$ https://wws.princeton.edu/sites/default/files/content/Social\%20Impact\%20Bonds\%202014\%20Final\%20Report.pdf

Berndt, C., \& Wirth, M. (2018). Market, metrics, morals: The Social Impact Bond as an emerging social policy instrument. Geoforum, 90, 27-35.

Brandstetter, L., \& Lehner, O. M. (2015). Opening the market for impact investments: The need for adapted portfolio tools. Entrepreneurship Research Journal, 5(2), 87-107.

Bridges Ventures. (2015). Ways to wellness SIB. Retrieved from https://www.bigsocietycapital.com/file/1639/download?token=CqYjHZf_ 
Carè, R. (2018). Social Impact Bond: Beyond financial innovation. In K. Wendt (Ed), Sustainable financial innovation (pp.19-171). London: Taylor \& Francis.

Center for Disease and Control prevention (CDC). (2017). Pay for success: How-to guide for local government focused on lead-safe homes. Retrieved from https://www.cdc.gov/nceh/lead/docs/pay_for_success_guide.pdf

Clay, R. F. (2013). Health Impact Bonds: Will investors pay for intervention? Environmental Health Perspectives, $121(2)$, a45.

Clifford, J., \& Jung, T. (2016). Social Impact Bonds: Exploring and understanding an emerging funding approach. In O. Lehner (Ed), Routledge handbook of social and sustainable finance (pp.161-175). London: Taylor \& Francis.

Commissioning Better Outcomes Fund Evaluation (CBOFE). (2014). Ways to wellness Social Impact Bond: The UK's first health SIB. Ways to wellness deep dive report. Retrieved from https://www.biglotteryfund.org.uk/-/media/Files/Programme\%20Documents/Commissioning\%20Better\%20Outcomes/CBO_ ways_to_wellness_report.pdf

Commissioning Better Outcomes Fund Evaluation (CBOFE). (2016a). Update report. Retrieved from https://www.biglotteryfund.org.uk/-/media/Files/Programme\%20Documents/Commissioning\%20Better\%20Outcomes/CBO \%20Update\%20Report_Full\%20Report.pdf

Commissioning Better Outcomes Fund Evaluation (CBOFE). (2016b). Mental Health and Employment Partnership (MHEP). In depth review. Retrieved from https://www.biglotteryfund.org.uk/-/media/Files/Programme\%20Documents/Commissioning\%20Better\%20Outcomes/comis sioning_better_outcomes_in_depth_review.pdf

Cooper, C., Graham, C., \& Himick, D. (2016). Social Impact Bonds: The securitization of the homeless. Accounting, Organizations and Society, 55, 63-82.

Corporation for National and Community Service (CNCS). (2016). State of the pay for success field II: Emerging literature, updates, $\quad$ and tools. $\quad$ Retrieved from https://www.nationalservice.gov/sites/default/files/documents/FR_PFS_Emergent\%20Literature\%20Review_2016_0.pdf

Cox, B. R. (2011). Financing homelessness prevention programs with social impact bonds. Review of Banking \& Financial Law, 31, 959-985.

Dear, A., Helbitz, A., Khare, R., Lotan, R., Newman, J., Sims, G. C., \& Zaroulis, A. (2016). Social Impact Bonds: The early years, 2016. Social Finance UK.

Development Impact Institute for Development Studies. (2015). Retrieved from http://opendocs.ids.ac.uk/opendocs/bitstream/handle/123456789/6820/Impact_investments_a_literature_review.pdf?seque nce $=1$

Dey, C., \& Gibbon, J. (2018). New development: Private finance over public good? Questioning the value of impact bonds. Public Money \& Management, 38(5), 375-378.

Dowling, E. (2017). In the wake of austerity: Social Impact Bonds and the financialisation of the welfare state in Britain. New Political Economy, 22(3), 294-310.

Edmiston, D., \& Nicholls, A. (2018). Social Impact Bonds: The role of private capital in outcome-based commissioning. Journal of Social Policy, 47(1), 57-76.

Emerson, J. (2012). Risk, return and impact: Understanding diversification and performance within an impact investing portfolio. Retrieved from http://www.impactassets.org/files/downloads/ImpactAssets_IssueBriefs_2.pdf

Fitzgerald, J. L. (2013). Social Impact Bonds and their application to preventive health. Australian Health Review, 37(2), $199-204$. Flynn, J., Young, J., \& Barnett, C. (2015). Impact investments: A literature review. CDI Paper. Brighton, United Kingdon: IDS.

Fox, C., \& Albertson, K. (2012). Is payment by results the most efficient way to address the challenges faced by the criminal justice sector? Probation Journal, 59(4), 355-373.

Fraser, A., Tan, S., Lagarde, M., \& Mays, N. (2018). Narratives of promise, narratives of caution: A review of the literature on Social Impact Bonds. Social Policy \& Administration, 52(1), 4-28.

Galloway, I. (2014). Using pay-for-success to increase investment in the nonmedical determinants of health. Health Affairs, 33(11), 1897-1904.

Global Humanitarian Assistance (GHA). (2017). Global humanitarian assistance-Report 2017. Retrieved from http://devinit.org/wp-content/uploads/2017/06/GHA-Report-2017-Full-report.pdf

Glynn, J. J., \& Murphy, M. P. (1996). Public management: Failing accountabilities and failing performance review. International Journal of Public Sector Management, 9(5/6), 125-137.

Golden, M. (2014). Pay-for-success financing: A new vehicle for improving population health? Pop Health News, 1(1), 1-3. 
Government Outcomes Lab (GO Lab). (2017). Better commissioning for healthy lives. A summary report. Retrieved from https://golab.bsg.ox.ac.uk/documents/120/GO_Lab_Better_Commissioning_for_Healthy_Lives_Symposium_-_Final.pdf

Grimsey, D., \& Lewis, M. K. (2005, December). Are public private partnerships value for money? Evaluating alternative approaches and comparing academic and practitioner views. Accounting Forum. Elsevier, 29(4), 345-378.

Gustafsson-Wright, E., Gardiner, S., \& Putcha, V. (2015). The potential and limitations of impact bonds: Lessons from the first five years of experience worldwide. Brookings: Global Economy and Development.

Institute for Child Success (ICS). (2016). Pay for success in the US. Summaries of financed projects. Retrieved from http://pfs.instituteforchildsuccess.org/wp-content/uploads/2016/06/ICS-4527-Summary-of-Pay-for-Success-Social-Impact-B onds-Paginated-October-2016.pdf

Iovan, S., \& Lantz, P. M. (2018). Social Impact Bonds: A promising public-private partnership model for public health. American Journal of Public Health, 108(8), e6-e6.

Jackson, E. T. (2013). Evaluating Social Impact Bonds: Questions, challenges, innovations, and possibilities in measuring outcomes in impact investing. Community Development, 44(5), 608-616.

Joy, M., \& Shields, J. (2013). Social Impact Bonds: The next phase of third sector marketization? Canadian Journal of Nonprofit and Social Economy Research, 4(2), 39-55.

Karanikolos, M., Mladovsky, P., Cylus, J., Thomson, S., Basu, S., Stuckler, D., ... \& McKee, M. (2013). Financial crisis, austerity, and health in Europe. The Lancet, 381(9874), 1323-1331.

Katz, A. S., Brisbois, B., Zerger, S., \& Hwang, S. W. (2018). Social Impact Bonds as a funding method for health and social programs: Potential areas of concern. American Journal of Public Health, 108(2), 210-215.

Keegan, C., Thomas, S., Normand, C., \& Portela, C. (2013). Measuring recession severity and its impact on healthcare expenditure. International Journal of Health Care Finance and Economics, 13(2), 139-155.

Kentikelenis, A. E., Stubbs, T. H., \& King, L. P. (2015). Structural adjustment and public spending on health: Evidence from IMF programs in low-income countries. Social Science \& Medicine, 126, 169-176.

Martin-Moreno, J. M., Anttila, A., von Karsa, L., Alfonso-Sanchez, J. L., \& Gorgojo, L. (2012). Cancer screening and health system resilience: Keys to protecting and bolstering preventive services during a financial crisis. European Journal of Cancer, 48(14), 2212-2218.

McDaid, D., La Park, A., \& Luis Fernandez, J. (2016). Reconnections evaluation interim report. Retrieved from https://golab.bsg.ox.ac.uk/knowledge/resources/reconnections-evaluation-interim-report/

McHugh, N., Sinclair, S., Roy, M., Huckfield, L., \& Donaldson, C. (2013). Social Impact Bonds: A wolf in sheep’s clothing? Journal of Poverty and Social Justice, 21(3), 247-257.

Monnet, N., \& Panizza, U. (2017). A note on the economics of philanthropy (No. 19-2017). Economics Section, The Graduate Institute of International Studies.

Moran, R. S., Moran, D. S., \& Fire, G. (2018). Social Impact Bonds for health promotion and preventive medicine. Harefuah, 157(1), 24-27.

Nazari Chamaki, F., Jenkins, G. P., \& Hashemi, M. (2018). Social Impact Bonds: Implementation, evaluation, and monitoring. International Journal of Public Administration, 42(4), 289-297.

Ng, A., Mirakhor, A., \& Ibrahim, M. H. (2015). Risk sharing and social impact partnerships. In Social capital and risk sharing (pp. 133-156). US: Palgrave Macmillan.

Nicholls, A., \& Tomkinson, E. (2013). The Peterborough Pilot Social Impact Bond. University of Oxford: Saïd Business School.

Normile, B., VanLandeghem, K., \& King, A. (2017). Medicaid financing of home visiting services for women, children, and their families. NASHP, August. Retrieved from https://nashp.org/wp-content/uploads/2017/09/Home-Visiting-Brief.pdf

OECD. (2016). Understanding Social Impact Bond. $\quad$ Retrieved from http://www.oecd.org/cfe/leed/UnderstandingSIBsLux-WorkingPaper.pdf

Reeder, N., Colantonio, A., Loder, J., \& Rocyn Jones, G. (2015). Measuring impact in impact investing: An analysis of the predominant strength that is also its greatest weakness. Journal of Sustainable Finance \& Investment, 5(3), 136-154.

Rizzello, A., Caré, R., Migliazza, M. C., \& Trotta, A. (2016). Social Impact Investing: A model and research agenda. In O. Lehner, (Ed.), Routledge handbook of social and sustainable finance (pp. 102-124). London: Taylor \& Francis.

Rizzello, A., \& Carè, R. (2016). Insight into the Social Impact Bond market: An analysis of investors. ACRN Oxford Journal of Finance and Risk Perspectives, 5, 145-171.

Rizzello, A., Caridà, R., Trotta, A., Ferraro, G., \& Carè, R. (2018). The use of payment by results in healthcare: A review and proposal. In Social impact investing beyond the SIB (pp. 69-113). Cham: Palgrave Macmillan. 
Rowe, R., \& Stephenson, N. (2016). Speculating on health: Public health meets finance in "Health Impact Bonds". Sociology of Health \& Illness, 38(8), 1203-1216.

Roy, M. J., McHugh, N., \& Sinclair, S. (2017). 14. Social Impact Bonds-Evidence-based policy or ideology? In Handbook of social policy evaluation (pp. 263-276). Cheltenham: Edward Elgar Publishing.

Rudd, T., Nicoletti, E., Misner, K., \& Bonsu, J. (2013). Financing promising evidence-based programs: Early lessons from the New York City Social Impact Bond. MDRC. https://www.mdrc.org/publication/financing-promising-evidence-based-programs

Saltuk, Y., Bouri, A., Mudaliar, A., \& Pease, M. (2013). Perspectives on progress: The impact investor survey. Global Social Finance, JP Morgan and the Global Impact Investing Network, London, January, 7. Retrieved from: https://www.jpmorganchase.com/corporate/socialfinance/document/207350_JPM_Perspectives_on_Progress_2013-01-07_10 18749_ada.pdf

Saltuk, Y. (2012). A portfolio approach to impact investment: A practical guide to building, analyzing and managing a portfolio of $\begin{array}{llll}\text { impact } \quad \text { investments. } & \text { Morgan. } & \text { Retrieved } & \text { from }\end{array}$ https://www.jpmorganchase.com/corporate/socialfinance/document/121001_A_Portfolio_Approach_to_Impact_Investment.p df

Schinckus, C. (2017). Financial innovation as a potential force for a positive social change: The challenging future of social impact bonds. Research in International Business and Finance, 39, 727-736.

Schinckus, C. (2018). The valuation of social impact bonds: An introductory perspective with the Peterborough SIB. Research in International Business and Finance, 45, 1-6.

Scognamiglio, E., Di Lorenzo, E., Sibillo, M., \& Trotta, A. (2018). Social uncertainty evaluation in Social Impact Bonds: Review and framework. Research in International Business and Finance, 47, 40-56.

Siemiatycki, M., \& Farooqi, N. (2012). Value for money and risk in public-private partnerships: Evaluating the evidence. Journal of the American Planning Association, 78(3), 286-299.

Sinclair, S., McHugh, N., Huckfield, L., Roy, M., \& Donaldson, C. (2014). Social Impact Bonds: Shifting the boundaries of citizenship. Social Policy Review, 26, 119-136.

Social Finance UK. (2018). Impact Bond global database. Retrieved from http://www.socialfinance.org.uk/database/

Tan, S., Fraser, A., Giacomantonio, C., Kruithof, K., Sim, M., Lagarde, M., ... \& Mays, N. (2015). An evaluation of Social Impact Bonds in health and social care: Interim report. Policy Innovation Research Unit (PIRU). Retrieved from https://piru.lshtm.ac.uk/assets/files/Trailblazer\%20SIBs\%20interim\%20report\%20March\%202015,\%20for\%20publication\% 20on\%20PIRU\%20siteapril\%20amendedpdf11may.pdf

Trotta, A., Caré, R., Severino, R., Migliazza, M. C., \& Rizzello, A. (2015). Mobilizing private finance for public good: Challenges and opportunities of Social impact bonds. European Scientific Journal, ESJ, 11(10), 259-279.

Warner, M. E. (2013). Private finance for public goods: Social Impact Bonds. Journal of Economic Policy Reform, 16(4), 303-319.

Weibel, A., Rost, K., \& Osterloh, M. (2009). Pay for performance in the public sector-Benefits and (hidden) costs. Journal of Public Administration Research and Theory, 20(2), 387-412.

Wilson, K. E., Silva, F., \& Ricardson, D. (2015). Social impact investment: Building the evidence base. Organisation for Economic Co-operation and Development. Retrieved from http://papers.ssrn.com/sol3/papers.cfm?abstract_id=2562082

Wise, A. (2017). A health outcomes fund for Canada. How paying for outcomes could improve health and deliver better value for money. MaRS Centre for Impact $\quad$ Investing. $\quad$ Retrieved from https://www.marsdd.com/wp-content/uploads/2017/02/A-Health-Outcomes-Fund-for-Canada.pdf 